\title{
Brazilian community health agents and qualitative primary healthcare information
}

\author{
Margareth S. Zanchetta ${ }^{1}$, Rogério Meireles Pinto ${ }^{2}$, Wilson Galhego-Garcia ${ }^{3}$, Zeilma da Cunha ${ }^{4}$, \\ Hésio A. Cordeiro ${ }^{5}$, Francisco E. Fagundes-Filho ${ }^{6}$, Mônica A.L. Pinhoo ${ }^{7}$, Susan M.V. Voet ${ }^{6}$, Yves Talbot $^{8}$, \\ Rodrigo S. Caldas ${ }^{9}$, Thiago J. de Souza ${ }^{9}$ and Edwaldo Costa ${ }^{9}$
}

\author{
${ }^{1}$ Daphne Cockwell School of Nursing, Faculty of Community Services, Ryerson University, Toronto, Canada \\ ${ }^{2}$ School of Social Work, Columbia University, New York, USA \\ ${ }^{3}$ Department of Basic Sciences, Faculty of Dentistry of Araçatuba, Estadual Paulista University, Araçatuba, São Paulo, Brazil \\ ${ }^{4}$ Masters in Family Health Program, Estácio de Sá University, Rio de Janeiro, RJ, Brazil \\ ${ }^{5}$ Masters in Family Health Program, Estácio de Sá University, Rio de Janeiro, RJ, Brazil \\ ${ }^{6}$ Independent Researcher, Toronto, Canada \\ ${ }^{7}$ Souza Marques Tecno-Educational Foundation \& Adolescentro Paulo Freire (Municipal Secretary of Healht and Civil \\ Defense of Rio de Janeiro), Rio de Janeiro, Brazil \\ ${ }^{8}$ Department of Family and Community Medicine, Faculty of Medicine \& Dala Lana School of Public Health, Health Policy \\ Management and Evaluation, University of Toronto, Toronto, Canada \\ ${ }^{9}$ Department of Basic Sciences, Faculty of Dentistry of Araçatuba, Estadual Paulista University, Araçatuba, São Paulo, Brazil
}

\begin{abstract}
Aim: The aim of this study was to explore female community health agents' views about the value of recording qualitative information on contextual health issues they observe during home visits, data that are not officially required to be documented for the Brazilian System of Primary Healthcare Information. Background: The study was conducted in community primary healthcare centres located in the cities of Araçatuba and Coroados (state of São Paulo) and Rio de Janeiro (state of Rio de Janeiro), Brazil. Methods: The design was a qualitative, exploratory study. The purposeful sampling criteria were being female, with a minimum of three years of continuous service in the same location. Data collection with 62 participants was conducted via 11 focus groups (in 2007 and 2008). Audio files were transcribed and submitted to the method of thematic analysis. Four themes guided the analysis: working with qualitative information and undocumented observation; reflecting on qualitative information; integrating/analysing quantitative and qualitative information; and information-sharing with agents and family health teams. In 2010, 25 community health agents verified the final interpretation of the findings. Findings: Participants valued the recording of qualitative, contextual information to expand understanding of primary healthcare issues and as an indicator of clients' improved health behaviour and health literacy. While participants initiated the recording of additional health information, they generally did not inform the family health team about these findings. They perceived that team members devalued this type of information by considering it a reflection of the clientele's social conditions or problems beyond the scope of medical concerns. Documentation of qualitative evidence can account for the effectiveness of health education in two ways: by improving preventative care, and by amplifying the voices of underprivileged clients who live in poverty to ensure the most appropriate and best quality primary healthcare for them.
\end{abstract}

Key words: Brazil; community health agents; deprived communities; information system; primary healthcare; qualitative health information; Rio de Janeiro; São Paulo

Received 6 October 2013; revised 23 March 2014; accepted 30 March 2014; first published online 25 April 2014

Correspondence to: Margareth S. Zanchetta, Daphne Cockwell School of Nursing, Faculty of Community Services, Ryerson University, Toronto, Canada. 350 Victoria St. office POD 468E, Toronto, Canada M5B 2K3. Email: mzanchet@ryerson.ca; margareth.zanchetta@yahoo.ca

(C) Cambridge University Press 2014 


\section{Introduction}

Brazil's Family Health Strategy (FHS) purports to use health education in primary healthcare (PHC) as a key strategy for improving the population's health awareness by providing individuals and families with information that may help them both prevent and treat myriad diseases and conditions (Cordeiro, 2005). Health information in Brazil's FHS is imparted by community health agents (CHAs) who provide information on an as-needed basis (Pinto et al., 2012). The paucity of literature on such educative work under conditions of social inequities that diminish the efficacy of PHC (Zanchetta et al., 2012) corroborates the need of investigating the clientele's disclosure of their varied, subjective perspectives of their health experience, as well as CHAs' experiential knowledge; the value of this type of evidence-based information has not been credited, but it can be highly useful in guiding health services planning and evaluation. In this study, we assumed that such additional information could be added to the system of quantitative epidemiological data to build a clearer portrait of service gaps that need to be addressed by multi-sectorial health promotion interventions, a key component of effective health promotion (World Health Organization, n/a).

We defined relevant qualitative information as that related to the clientele's experiential, behavioural and affective issues of well-being, revealing information about social, economic, and political structures that are not documented in the current health information registry as implemented in Brazil's PHC system. Using a health promotion lens, such qualitative information draws on the social determinants of health to indicate areas where expected outcomes of FHS could be potentially undermined. Consequently, we explored the type of qualitative information that triggered CHAs' attention and decision to document their clients' comments and behaviour that was pertinent to the course of CHAs' and FHS team's professional actions.

Effects of CHAs' work were found as being the moderate positive changes in maternal-child health indicators, low positive impact on health indicators in terms of reduced infectious diseases and low positive changes in indicators of reduced social inequities (Giugliani et al., 2011). There is no evidence on the types of qualitative information (facts, situations, and personal accounts) CHAs gather from community clients and how they reflect upon and share this information with FHS teams to help clients deal with contextual social determinants of their health.

This study thus aimed to highlight CHAs' subjective experience of valuing and using his kind of qualitative information in their practice to uncover their clients' life context. The importance of this study concerns the identification of a synergism among multiple socio-ecological, non-measurable indicators as emergent beneficial features in the work of CHAs for whom there are no formalized tools for practice.

\section{Background and context}

According to Zanchetta et al. (2009), CHAs are officially recognized by law as health professionals. Their work setting includes a community primary healthcare centre, where an array of PHC and secondary healthcare services is free and accessible to the local population as part of the national official health program. It is customary in CHAs' day-today practice to share with community clients their own life story and experience of social ascension, to act as role models of personal empowerment and to help clients understand health-related information (Zanchetta et al., 2005; Côtê et al., 2010), as well as provide educational health support to help clients to incorporate medical and health information in a manner that is in harmony with existing popular health knowledge (Zanchetta et al., 2012).

The FHS continuously monitors health indicators that are tracked by the Integrated System of Basic Information (SIAB), the Ministry of Health's computerized system for assembling and managing health-related information. This information is used to determine government funding levels allocated through municipal secretaries of health (Brazil, Ministério da Saúde, 2005). CHAs manually collect information filling in standardized forms that are limited to specific socio-demographic and epidemiological data. To date, no behavioural data is included, and the tools proposed to evaluate the SIAB (Brazil, Ministério da Saúde, 2005), do not consider behavioural data.

When this study was conducted, almost 180000 CHAs composed the workforce: $81 \%$ of them were women, and $67 \%$ of them were under the age 
of 34. This study was conducted with CHAs whose socio-demographic characteristics correspond to the national profile of female CHAs: most have professional experience, are younger than 35 years old, receive a minimal salary [as of 2007, 540 reais (US\$ 324) per month], have formal education that ranges from incomplete elementary school to high school, and work predominantly in urban settings (Brazil, Ministério da Saúde, n/a).

\section{Conceptual framework}

Bourdieu's theoretical concept of habitus guided this study. Bourdieu (1986) developed a theory of culture in which individuals occupy a multidimensional space (grounded in forms of economic, cultural, and social capital) which reproduces inequality and yields a sense of one's place in a social hierarchy. Habitus is formed through multiple engagements in the social world and includes embodied and unconscious practices. Héran (1987) affirms that habitus refers to more than a habitual, passive way of being since it relates to an individual's story and his/her discourse that exteriorizes what was interiorized as a form of available capital. Habitus embodies a core idea of 'being' and 'have being', leading the individual in their decisions, choice, judgement, etc. As a product of individual and collective history, habitus leads to an organizing action, a way of being, as well as a storage of past experiences towards a predisposition for the future.

With the concept of habitus in mind, we also incorporated into the study design and analysis various feminist concepts about women's roles (eg, learnt, assigned or socially expected), ways of knowing, caring for others, as well as relational, political, and affective aspects of participation in the workforce (Armstrong and Armstrong, 1990; Wood, 1994; Belendy et al., 1997; Loutfi, 2001; Lips, 2006). We applied these concepts to guide the study design and analysis strategies to assess the social, daily work of CHAs who practice as professionals in social contexts that are the same or similar to the ones in which they were born, raised, and lived or still live.

\section{Research questions}

(1) Which facts, situations, and personal accounts about their clients caught the attention of CHAs?
(2) What kind of reflections resulted from the information gleaned through observations made during home visits? (3) How did the CHAs integrate and analyse the quantitative data with the qualitative information? (4) What kind of information did the CHAs share among themselves and with FHS professionals?

\section{Methods}

\section{Design and data collection}

This qualitative, exploratory study was conducted in community primary healthcare centers (CPHCC) located in the following settings and municipalities: a major compound of five deprived communities in a metropolis (the neighbourhood of Tijuca in the city of Rio de Janeiro), a poor neighbourhood (named São José), a small deprived community in a middle-size city (Araçatuba), and a rural area (a vast zone in Coroados municipality), the latter all in the state of São Paulo.

For the sampling strategy, CHAs' supervisors pre-screened prospective participants based on the inclusion criteria: female gender, with a minimum of three years of continuous service as a $\mathrm{CHA}$ in the same CPHCC. Then a study presentation letter was sent to all prospective participants, with a recommendation to expect further information from their supervisor about participation in the study. Sixty-two CHAs contacted their supervisors expressing their interest in participating, and constituted the final sample group. We did not track the number of non-responders to the invitation, but the final sample size exceeded our initial expectation of having a sample of 15-21 participants.

\section{Data collection}

Data collection sessions took place in the CPHCC facilities, with no involvement by the professional and administrative staff. Since the CHAs had never participated in a study before, special attention was given by the local principal investigators (PIs) (W.G.G. and Z.C.) to explaining the study and obtaining signed informed consent. At the beginning of each data collection session, the consent form was read aloud by the local PIs to study participants; about $15 \mathrm{~min}$ was spent reading and reviewing the terms of consent and answering participants' questions. Emphasis was placed on issues of confidentiality 
Table 1 Guide for focus group discussion

Questions

Which client situations, behaviours, or stories attract your attention during your home visits with your clients?

Which of these sorts of information do you share with the FHS team? Which sorts do you not share with them? Why?

How do you know that something different is happening with your client's health?

During the home visit and after having observed and collected information from the family, what information do you usually think is important and valuable to pass on regarding the problems they told you about?

How do you relate the information you gather from clients during home visits with the clinical information you collect to record on the SIAB form?

What kind of client information do community health agents share among themselves?

What significance do you give to the information you collect by observing clients during home visits?

and CHAs' right to cease their voluntary, unpaid participation without compromising their professional activities or relationships with supervisors and researchers. All participants signed the consent form and received a copy for their personal files.

Data collection by local PIs and their research assistants (RAs) included single-session focus group discussions lasting two hours on average and were audio recorded [three focus groups were held with a total of 17 CHAs in Rio de Janeiro (in August 2008); seven focus groups were held with 38 CHAs in Araçatuba (in June-August 2007); and one focus group was held with seven CHAs in Coroados (in August 2007)]. Local RAs were responsible for monitoring and audiorecording the sessions and taking notes on the proceedings. All documentation and audio recordings (in CD-ROM format) remained under the responsibility of the lead PI for a period of five years. As our focus was to uncover CHAs' views about qualitative health information and we were aware of a lack of any documentation on this by the CPHCC, this was the only source of data gathered in the initial phase of the study.

\section{Interview protocol}

The investigation team created a guide of semistructured questions (see Table 1) that were used to guide focus groups with CHAs. The discussion was based on the CHAs' praxis and the use of observations made and recorded during home visits. It reflected the empirical documentation of habitus in the work of women as CHAs in that the questions aimed to provoke verbalization of their ways of perceiving, reflecting and acting upon the social context of their praxis. No CHAs provided any feedback on the questions, but the questions were appraised by local PIs with the collaboration of FHS supervisors. No pilot testing was done.

\section{Addittional empirical evidences}

To gain a broader context for understanding what we heard from CHAs and as a way to corroborate the data, we held meetings with four FHS managers (a dentist, a lawyer, a nurse and a physician) in each study site. Exploring managers' points of view allowed us to understand the macroperspective of CHAs' practice and its structural context. It was beneficial to detect other perspectives from FHS teams, including political constraints. Six months later, two additional group discussions of 90 min each were held with FHS managers at each study site to obtain their views on CHAs' practice and the value of recording qualitative data. These meetings were audiorecorded by the lead PI to allow for further analysis and checking of the data. The digital audio files were transcribed and reviewed by the RAs and the main ideas were manually retrieved by the lead PI. After that, the audiofiles were deleted. It is noteworthy that the site managers in Araçatuba decided to have their meetings videorecorded by local RAs and to document some of the discussion for their own use, to animate debate on the theme among FHS professionals and faculty.

\section{Data analysis}

Focus groups were audio recorded and transcribed by the RAs, followed by a one-time discussion with the local PIs about the RAs' immediate thoughts after conducting the focus groups. As transcripts were completed, they were read by all co-investigators and discussed among 
the lead PI, two Toronto-based co-investigators and the PI for Araçatuba (in Toronto during March 2008), Rio de Janeiro's team with the lead PI (in November 2008 and May 2009) and Araçatuba's team with the lead PI (in December 2008). All the researchers closely examined their understandings of the CHAs' accounts, and the national and foreign researchers worked together to identify their own beliefs, knowledge and assumptions about CHAs' social and personal knowledge. The common ground of experience with PHC in Brazil promoted harmony in resolving any divergent points of view.

RAs and local PIs met the lead PI for an initial discussion on data analysis. The transcriptions were coded by the lead PI using the software Atlas ti and analysed using the qualitative method of thematic analysis (Paillé and Mucchielli, 2008). The thematic analysis followed these procedures: (a) a preliminary list of codes was developed using the research questions and aims, as well as the focus group discussion questions as the initial framework, (b) themes were pre-defined after repeated readings of the texts, (c) a thematic log was created, with reflections about discussion content and attempts to group themes as part of the inductive first level analytical process, documented as memos in the software, (d) the transcribed texts were then coded, producing an output of verbatim discourse that was arranged according to these pre-defined themes: (1) working with qualitative information and undocumented observations; (2) reflecting on qualitative information; (3) integrating/analysing quantitative and qualitative information, and (4) information-sharing among CHAs and with the FHS team; (e) the pre-defined theme labels were then confirmed, reflecting the scope of the analysed transcripts, and (f) the research questions were answered using the themes as a final framework to determine the results based on the descriptive and analytical texts.

To ensure analytic rigour, the lead PI shared the final analytical product with the local PIs to avoid bias and selectivity in the findings. Local PIs integrated their own reflections and interpretations as well as that of their RAs regarding their first impressions and thoughts immediately after data collection sessions. An additional two meetings with another 22 volunteer $\mathrm{CHAs}$ were held by the local PIs in Araçatuba (in May 2010) and with three volunteer CHAs in Rio de Janeiro (in November 2010) for member checking. The volunteer groups verified the final inter- pretation of the findings and ensured their trustworthiness (Lincoln and Guba, 1999). All the CHAs corroborated the summary interpretation of the data.

\section{Findings}

This section presents the views of 62 female CHAs on the scope of importance they gave to incorporating qualitative information to underpin the success, efficacy, and quality of health promotion activities. Their socio-demographic characteristics follow: in Araçatuba, 38 CHAs were aged 34-46 years old, married, had a high school level of education and three to six years of professional experience in shantytowns and poor neighbourhoods; in Coroados, seven CHAs were aged 37-48 years old, married or single, had an elementary level of education and six years of professional experience in rural areas and poor neighbourhoods; in Rio de Janeiro, 17 CHAs were aged 23-52 years old, married or single, had a high school level of education and three to 10 years of professional experience in shantytowns and poor neighbourhoods. The context of practice involved mainly PHC through educative interventions during home visits and other forms of follow-up in schools, kindergarten and community daycare for children and adults, and eventually other places of community assembly (ie, bars, restaurants, gardens, and churches). The total numbers of CHAs and their client base per site were: 39 CHAs and 11500 clients in Rio de Janeiro; 196 CHAs and 113400 clients in Araçatuba; and 11 CHAs and 4417 clients in Coroados.

The results are presented in the next sections, organized according to the analytical themes and in response to the research questions. Verbatim excerpts illustrate some of the CHAs' accounts and are identified solely by their city of origin.

\section{Working with qualitative information and undocumented observations}

Regarding the facts, situations, and personal accounts about CHAs' clients that caught their attention during home visits, all the CHAs reported that while the traditional resistance to change in deprived communities was a jeopardizing factor in their educative work, it was common to promote 
behavioural change by getting clients to adopt a new practice; this was the case in all the geographic locations and practice settings. CHAs used persuasive arguments and cajoling to motivate clients and their relatives to act:

You said that you adore your mom but if you do not go to a doctor with her... well, [you] better kiss her for the last time because she won't be able to live much longer with such high blood sugar.

\section{(CHA, Rio de Janeiro)}

Even those CHAs working in a deprived community in Rio de Janeiro, where violence is common and lease to the destruction of social bonds, have witnessed behavioural change based on an increasing general awareness about quality of life issues. The local population has gradually expressed more cordiality towards CHAs and the work of CHAs has received more recognition, mainly by community organizations. CHAs cited the following positive changes in client behaviour: increased adherence to proposed plans of care, acceptance of referrals, actively searching for healthcare, and self-monitoring of clinical conditions.

Much of the qualitative information noted by CHAs related to family abuse and violence, especially against children and seniors (including financial extortion):

A lady 70 years old, single.... Her neighbour learned that she had a retirement pension. She lived with him for a while, and then he sold her house and sent her to the streets.

(CHA, Rio de Janeiro)

Other observations indicated manifestations of a client's emotional troubles (outbursts of anger, disrupted sleep patterns, anxiety, home confinement), as well as their family's interpersonal problems.

We broke into that house because there were two children locked up for almost three days... tied up with no food.... An auntie took care of them after [they were] released by the Children Protection Services.

(CHA, Rio de Janeiro)

Usually, CHAs detected such situations by observation of the home environment and the family's social dynamics. They never accepted the 'apparent state of everything being alright' and always compared previous information with the most recent. Sometimes CHAs detected that 'something [was] wrong' at the start of a home visit. This immediate insight was intuitive and related as, 'I do not know [how I knew]... [I had] a kind of a clue', and a CHA would elaborate her suspicions and hypotheses which were immediately or later corroborated when talking to her client. By this type of sensitive attention and probing, CHAs ensured congruency between their observations and the client's complaints or concerns.

CHAs tended to concentrate on some 'foci' of information that the client provided. Sometimes it was a 'lack of clues' about the reported facts that justified the additional collection of information. CHAs in Araçatuba and Coroados emphasized that close contact with their clients made observation easier. Clues such as an unusual facial expression, disturbing eye contact or alteration in voice intonation triggered the CHAs' 'sharp intuition' to uncover the existence of a problem. CHAs stressed the value of their freedom to probe and gradually appraise a situation by engaging a client in open dialogue and colloquial chat. Such an informal approach was perceived to be more effective, made possible by the friendly relations between CHAs and their clients. It is noteworthy that the development of friendships between CHAs and their clients is considered socially acceptable since most of them belong to the same community. Despite the lack of a Code of Ethics for CHAs regarding professional boundaries, in the Brazilian sociocultural context, compassionate, even intimate, communication between professionals and clients is perceived as an appropriate element of personalized and humanized healthcare.

Sometimes I identify the problem by eye contact... and other times [the client] may welcome me... crying. Just by looking at the person, you already identify that she is not well.

(CHA, Araçatuba)

\section{Reflecting on qualitative information}

Regarding the kind of reflections associated with the information gleaned through observations made during home visits, CHAs remarked on the importance of developing social sensitivity to 
'see beyond' appearances and how that guided them to identify previously unnoticed problems. CHAs revealed that their interpersonal links facilitated dialogue with their clients, who, they were sure, would never lie to them. One CHA described how such a trusting relationship promoted a client's disclosure of a problem that she initially withheld:

[The client] said that she was [fine], but I saw in her face that she wasn't. Then she offered me a coffee and I accepted.... Then she said, 'Oh, as it's you, I must tell'.

(CHA, Araçatuba)

CHAs in Rio de Janeiro who saw the effects of poverty in deprived communities reflected on their observations and questioned the ethical implications of ignoring the socio-economic conditions in which their clients live. They considered it important to maintain awareness and sensitivity to their clients' circumstances and to integrate this in their daily context of work.

We collect information and we stay there listening for what we could someday use... to have a kind of weapon to defend [the client], or to give a shot of enthusiasm against the social pain.

(CHA, Rio de Janeiro)

Rio de Janeiro's CHAs were intimately aware of the deleterious effects of poverty and the lack of opportunities to improve life conditions. Their reflections extended to the effects of poverty on the psychological well-being of families and on their own professional responsibilities for providing preventative care.

\section{Integrating and analysing quantitative and qualitative information}

CHAs reported having some difficuly integrating quantitative data with the qualitative information they collected. According to CHAs, while the quantitative information they reported is intended to measure the incidence of disease, the qualitative information revealed other critical aspects of individual and community health. Therefore, CHAs developed the practice of keeping a hand-written $\log$ in a notebook where they documented their personal notes based on observations and reflections about issues that would require their follow-up and weekly or monthly monitoring. All the CHAs had a similar analysis and criticism about the narrow focus of the type of information the SIAB form covered:

The form does not ask [about the state of] the person's house, whether the person lacks any necessities, whether the person is illiterate...

(CHA, Araçatuba)

[The form counts] how many people have diabetes, hypertension... It does not [consider] the causes, just the quantity!

(CHA, Rio de Janeiro)

The form should be more complete [by including], for instance, house hygiene, schooling level, depression.

(CHA, Coroados)

CHAs denounced the fact that socio-economic issues and the social roots of medical problems were barely identified, and that the importance of these aspects remained devalued by FHS managers and other decision makers. CHAs in Rio de Janeiro referred to a portrait of health promotion initiatives based solely on the increased numbers of home visits or group activities for health education while the incidence of pregnant teens, work with parents' support groups, and drug addiction and so on was omitted.

Problems like drug use, no! That sort of information is not much detailed, but they are sent to SIAB.

(CHA, Coroados)

\section{Information sharing among CHAs and with the FHS team}

The kind of information that CHAs shared among themselves and with FHS professionals was a matter of personal choice. Few CHAs shared with each other the qualitative information they observed or collected; doing so depended on their understanding of the notion of ethics and trust. Note-taking remained an individual informal activity, and CHAs perceived sharing such 'not-to-be-documented 
information' a matter of confidentiality. As for sharing this information with FHS professionals, it depended on CHAs' certainty about how professionals would receive and deal with the information. Some CHAs engaged in information sharing among each other when they wanted to gain a better understanding of the underlying causes of clients' conditions, to obtain advice on how proceed, or to notify the nurse supervisor.

If you want, later you can pass it to the team to discuss, [but] usually we do not have any contact. We [may] talk about the situation, ask for an opinion.

\section{(CHA, Coroados)}

The main reason for not sharing information with FHS professionals was CHAs' conviction that physicians and nurses had an opposite vision about the importance of the information they noted. Moreover, CHAs perceived a lack of commitment and collaboration between FHS and CHA teams. Consequently, some CHAs were selective about what qualitative information they shared about the clinical cases. Some decided not to share information; others only discussed major social, economic or behavioural issues.

What is really shared is health problems like diabetes and hypertension, and we also talk about what happened in our work.

(CHA, Araçatuba)

\section{Meetings with FHS managers}

The corroboration of data was done by FHS managers who perceived the sort of qualitative information CHAs were concerned about as a matter of a population's social conditions. The managers revealed historical and political information on the functioning of the PHC system that corroborated and explained the basis for the CHAs' criticisms about systemic neglect of the social determinants of health.

The managers did not mention any result of CHAs' educative work regarding changes in their clients' health attitudes or behaviour. The managers gave priority to philosophical issues related to the FHS and critical reflections on SIAB. Managers from Araçatuba and Coroados emphasized that due to officials' concerns over giving CHAs freedom to address social issues, FHS managers historically have adopted a rigid standard and protocol for the SIAB recording system. As a result, the system contains repetitive questions that skew the information collected due to its pre-determined format for recording data. Consequently, FHS teams tend to analyse only data related to diseases of collective interest, basic sanitation and hygiene habits. For instance, Rio de Janeiro's manager revealed that the high level of community and gang violence produces spontaneous demand for services due to somatic manifestations such as anxiety, headache, and mental health issues. She disclosed that some psycho-social interventions (self-help groups, debriefing meetings) were implemented, but they only counted the number of held sessions, with no qualitative information about their impacts on clients' quality of life or mental health. A lack of administrative structure contributes to the absence of follow-up or measures of such outcomes.

\section{Discussion}

CHAs understood that their observations about their clients' home environment were distinct from the data officially accepted by FHS teams. By neglecting such situational information, FHS teams restrict the development of CHAs' technical competencies in promoting PHC (Adell et al., 2009). The lack of information sharing with FHS professionals indicates the need for education on ethical principles that would help CHAs to review their professional and personal reasoning about potentially inappropriate actions. Trust and the confidentiality of client information are also at stake. Some investigators (Abdalla and Nichiata, 2008; Seoane and Fortes, 2009) have found that both stigmatized diseases and gender differences between clients and CHAs tend to restrain clients' disclosure of personal concerns, even when there is no perceived risk to client confidentiality. It is important to note that in addition to the difficulties created by an inefficacious system of referral and counter-referral (Escorel et al., 2007), the lack of integration of health services and poor continuity of care puts those relying solely on community-level healthcare services at greater risk of poor health outcomes.

The CHAs' critical awareness of the significance of this rich observation was witnessed by the lead PI 
Table 2 Common critical issues in CHAs' accounts of qualitative information in their educative practice

Interpretation of empirical data from all study sites

Distorted self-perception of being a multi-task professional led CHAs to implicitly create the possibility of managing client referrals and counter-referrals to FHS teams.

Critical awareness that relevant qualitative information has been lost.

Social and professional roles are nebulous and $\mathrm{CHAs}^{\prime}$ personal vulnerability restrained the scope of their actions regarding the documentation of critical situations and denunciation to local authorities.

Frustration regarding their lack of education related to skills to identify and appraise the impact of important socioeconomic factors on community health.

Severe criticism of the use of overly-complicated language in their training and education. Instructing CHAs to use plain language would be more effective when inquiring about clients' health during fieldwork. Urgent need to help others led CHAs to become personally involved in social/family dramatic situations; this tendency was exacerbated by their social solidarity and distorted professional boundaries. Use of an 'informal' understanding of confidentiality and ethics undermined CHAs professional confidentiality and impaired information-sharing with FHS team.

Intensity of violence in the neighbourhoods determined the scope of $\mathrm{CHAs}^{\prime}$ use of personal power; their decision-making and authority challenged conventional views of gender differences and women's role.

during her previous contact with CHAs in Rio de Janeiro. CHAs are quite willing to help other health professionals who want to create an electronic format for registering daily life situations and cease the unproductive, limited reporting of diseases and environmental conditions. CHAs apply their communication skills, similar life experiences, sense of solidarity, and compassion to closely observe their clients, elicit their trust, and obtain a wealth of intimate, contextual information. An empathic manner of communication, perseverance, and expertise on lay knowledge constitute the CHAs' major skills (Pinto et al., 2012). CHAs insist on recording physical, material, and psychosocial factors as social determinants of health (Buss and Carvalho, 2007) that challenge their ability to promote health education, particularly in deprived communities. The finding that this information is not welcomed, used by or in disagreement with the health bureaucracy (Greenway et al., 2013) corroborates the devaluing of care provided by women as CHAs (George, 2008; Standing et al., 2008; Lynch and Walsh, 2009). The findings reveal CHAs' social sensitivity, solidarity and commitment to fight against social inequities and disparities as a matter of social justice that healthcare organizations can sustain (George, 2008; Pérez and Martinez, 2008). The CHAs' similar accounts of gathering qualitative information mainly through direct observation highlight their commonality in terms of social awareness, solidarity, roles, educative practice, training and practice context (see Table 2).

\section{Limitations}

While we did not directly observe the CHAs' practice in this study, we do not consider this a major methodological threat because five of the co-investigators possess vast experience as researchers and practitioners who are familiar with the practice of CHAs. Such expertise allows us to assert that the documentation of qualitative information would be a good practice for health promotion teams in other countries, especially those whose hierarchical health systems rely on front-line community workers who are themselves marginalized (Pérez and Martinez, 2008).

\section{Conclusion}

We believe that documentation of qualitative evidence of a population's progress in health practices would counter arguments about the lack of positive outcomes from the educative work of community health workers (Lewin et al., 2005). The role and contribution of such personnel to the health of vulnerable and hard-to-reach populations was recently recognized by the USA Community Health Workers Initiative (Ingram et al., 2012). We should implement innovative ways to document the effectiveness of health education, and use cost-effective methods to do so. Systematic observation guides can provide a model for new managerial tools to transform qualitative findings into numerical indicators. The use of such an information system in technological devices by community health workers is 
highly feasible in complex, hard-to-manage, equitable PHC systems in developing and less developed countries (Illyemi et al., 2007).

\section{Acknowledgements}

The authors gratefully acknowledge the enthusiastic participation of $62 \mathrm{CHAs}$ who shared their experiences with us, and those 25 CHAs who assisted us in the interpretation phase of the study. Many thanks to the CHAs' supervisors at Coordenadoria Geral de Saúde da Ârea Programática 2.2 (Prefeitura da Cidade do Rio de Janeiro), Centro de Saúde Três de Coroados, and Secretaria Municipal de Saúde de Araçatuba who facilitated the data collection sessions, offered rooms, and rearranged CHAs' work schedules to allow their participation in those sessions. Special thanks to four undergraduate medical students enrolled in Estácio de Sá University, trainees of the National Program of Scientific Initiation, and four undergraduate students of journalism and communication, research interns at Department of Basic Sciences, Faculty of Dentistry, Estadual Paulista University, who were volunteer RAs for this study, as well as to Dr Marilene Cabral do Nascimento who kindly supported the participation of undergraduate medical students (University Estácio de Sá) as well as, Dr Paulo Roberto VasconcellosSilva for reviewing an early draft of this paper. Many thanks to Sylvia Novac who edited the manuscript.

\section{Financial Support}

This study was funded by Association of Universities and Colleges of Canada, Canada-Latin America and the Caribbean Research Exchange Grants 2007 \& International Research Development Centre - Canada and Ryerson University, Faculty of Community Services 2013 Writing Week Initiative.

\section{Conflicts of Interest}

None.

\section{Ethical Standards}

This study was approved by Ryerson University Research Ethics Board, Rio de Janeiro Municipal

Primary Health Care Research \& Development 2015; 16: 235-245
Secretary of Health and Civil Defense- Ethics Committee, Estadual Paulista University, Ethics Committee and Brazil Research Ethics National Commission.

\section{References}

Abdalla, F.T.M. and Nichiata, L.Y.I. 2008: A abertura da privacidade e o sigilo das informações sobre o HIV/Aids das mulheres atendidas pelo Programa Saúde da Família no município de São Paulo, Brasil. Saúde \& Sociedade 17, 140-52.

Adell, C.N., Echevarria, C.R. and Bentz, R.M.B. 2009: Curso virtual para el desarrollo de competencias en atención primaria de salud. Revista Panamericana de Salud Publica 26, 176-83.

Armstrong, P. and Armstrong, H. 1990: Theorizing women's work. Toronto: Garamond.

Belendy, M.F., Clinchy, B.M. and Goldberger, N.R. 1997: Women's ways of knowing: the development of self-voice and mind. New York: Basic Books.

Bourdieu, P. 1986: The forms of capital. In Richardson J.E., editor, The handbook of theory: research for the sociology of education. Chapter 9. New York: Greenwood Press, 241-58.

Brazil, Ministério da Saúde. 2005: Avaliação para melhoria da qualidade da estratégia da família. Caderno de autoavaliação. Equipe Saúde da Família-Parte I. 4. (Evaluation of quality improvement of the Family Strategy. Booklet of self-evaluation. Family health team. Part I. 4). Brasilia, Brazil: MS.

Brazil, Ministério da Saúde. n/a: Secretaria da Gestão do Trabalho e Educação à Saúde. (Secretary of work management and health education). Retrieved 2 May 2007 from http://portal.saude.gov.br/portal/sgtes/visualizar_texto.cfm? idtxt $=23176$

Buss, P. and Carvalho, I.A. 2007: Health promotion in Brazil. Global Health Promotion 14, 209-13.

Cordeiro, H. 2005: SUS: Sistema Único de Saúde. (SUS: unified health system), second edition. Rio de Janeiro: Editora Rio.

Côtê, R., Renaud, L., Rhéaume, J. and Zanchetta, M. 2010: Retour de mission: visite au Brésil pour disséminer de l'information sur les déterminants sociaux de la santé et les vulnérabilités sociales. Cahiers METISS 5, 47-60.

Escorel, S., Giovanella, L., Mendonça, M.H.M. and de Castro Maia Senna, M. 2007: The family health program and the construction of a new model for primary care in Brazil. Revista Panamericana de Salud Publica 21, 164-76.

George, A. 2008: Nurses, community health workers, and home carers: gendered human resources compensating for skewed health systems. Global Public Health 3, 75-89.

Giugliani, C., Harzheim, E., Duncan, M.S. and Duncan, B.B. 2011: Effectiveness of the community health worker in Brazil: a systematic review. Journal of Ambulatory Care Management 34, 326-38. 
Greenway, J.C., Entwistle, V.A. and terMeulen, R. 2013: Ethical tensions associated with the promotion of public health policy in health visiting: a qualitative investigation of health visitors' views. Primary Health Care Research \& Development 14, 200-11.

Héran, F. 1987: La seconde nature de l'habitus. Tradition philosophique et sens commun dans le langage sociologique. Revue française de sociologie 28, 385-416.

Illyemi, A., Briggs, J. and Adams, C. 2007: Mobile information system, health work and community health workers in less developed countries. 16th IEEE International Workshops on Enabling Technologies: Infrastructure for Collaborative Enterprises (WETICE 2007), 208-212. Retrieved 2 August 2012 from http://www.journals1.scholarsportal. info.tmp/15364292804856178415.pdf

Ingram, M., Reinshmidt, K.M., Schachter, K.A., Davidson, C.L., Sabo, S.J., De Zapien, J.G. and Carvajal, S.C. 2012: Establishing a professional profile of community health workers: results from a national study of roles, activities and training. Journal of Community Health 37, 529-37.

Lewin, S.A., Dick, J., Pond, P., Zwarenstein, M., Aja, G., van Wyk, B., Bosch-Capblanch, X. and Patrick, M. 2005: Lay health workers in primary and community health care. The Cochrone Database of Systematic Reviews, 4. Retrieved 5 May 2007 from http://www.thecochranelibrary.com

Lincoln, Y. and Guba, E. 1999: Establishing trustworthiness. In Bryman, A. and Burgess, R.G., editors, Qualitative research. Chapter 11. Volume III, Thousand Oaks, CA: SAGE Publications, 289-331.

Lips, H. 2006: A new psychology of women: gender, culture and ethnicity, third edition. New York: McGraw Hill.

Loutfi, M.F. 2001: Women, gender and work: what is equality and how do we get there? Geneva: International Labour Office.

Lynch, K. and Walsh, J. 2009: Love, care and solidarity: what is and is not commodifiable. In Lynch, K., Baker, J. and Lyons, M., editors, Affective equality: love, care and injustice. Chapter X. United Kingdom: Palgrave Macmillan, 35-53.

Paillé, P. and Mucchielli, A. 2008: L'Analyse Qualitative en Sciences Humaines et Sociales. (Qualitative analysis in social and human sciences), second edition. Paris: Armand Colin.

Pérez, L.M. and Martinez, J. 2008: Community health workers: social justice and policy advocates for community health and well-being. American Journal of Public Health 98, 11-14.
Pinto, R.M., da Silva, S.B. and Soriano, R. 2012: Community health workers in Brazil's unified health system: a framework of their praxis and contributions to patient health. Social Science \& Medicine 74, 940-47.

Pinto, R.M., Wall, M., Yu, G., Penido, C. and Schmidt, C. 2012: Primary care and public health services integration in Brazil's Family Health Strategy. American Journal of Public Health 102, e69-76.

Seoane, A.F. and Fortes, P.A.C. 2009: A percepção do usuário do Programa Saúde da Família sobre a privacidade e a confidencialidade de suas informações. Saúde \& Sociedade $18,42-49$.

Standing, H., Mushtaque, A. and Chowdhury, R. 2008: Producing effective knowledge agents in a pluralistic environment: what future for community health workers? Social Science \& Medicine 66, 2096-2107.

Vasconcelos, E.M. 2007: Educação popular: instrumento de gestão participativa dos serviços de saúde. In Ministério da Saúde, Caderno de educação popular e saúde. (Handbook of popular education and health) Chapter 1. Brasilia: Ministério da Saúde, Secretaria de Gestão Estratégica e Participativa, Departamento de Apoio à Gestão Participativa, 19-30.

Wood, J. 1994: Who cares? Women, care and culture. Carbondale, IL: Southern Illinois University.

World Health Organization. (n/a): The Ottawa charter for health promotion. Retrieved 3 September 2012 from http:// www.who.int/healthpromotion/conferences/previous/ottawa/ en/index1.html

Zanchetta, M.S., Kolawole-Salami, B., Perreault, M. and Leite, L.C. 2012: Scientific and popular health knowledge in the education work of community health agents in Brazilian shantytowns. Health Education Research 27, 608-23.

Zanchetta, M.S., Leite, L.C., Perreault, M. and Lefebvre, H. 2005: Education and professional strengthening of the community health agent: an ethnography study. Online Brazilian Journal of Nursing. Retrieved 10 February 2008 from http://www.objnursing.uff.br/index.php/nursing/article/ view/35

Zanchetta, M.S., McCrae Vander Voet, S., Galhego-Garcia, W., Smolentzov, V.M., Talbot, Y., Riutort, M., Galhego, A.M., de Souza, T.J., Caldas, R.S., Costa, E., Kamikihara, M.M. and Smolentzov, S. 2009: Effectiveness of community health agents' actions in situations of social vulnerability. Health Education Research 24, 330-42. 